\title{
Nanoscale
}

Cite this: Nanoscale, 2013, 5, 9732

\section{A "nano-windmill" driven by a flux of water vapour: a comparison to the rotating ATPase $\dagger$}

\author{
Patrycja Nitoń, Andrzej Żywociński, ${ }^{\star}$ Marcin Fiałkowski and Robert Hołyst*
}

We measure the frequency of collective molecular precession as a function of temperature in the ferroelectric liquid crystalline monolayer at the water-air interface. This movement is driven by the unidirectional flux of evaporating water molecules. The collective rotation in the monolayer with angular velocities $\omega \sim 1 \mathrm{~s}^{-1}$ (at $T=312 \mathrm{~K}$ ) to $10^{-2} \mathrm{~s}^{-1}$ (at $T=285.8 \mathrm{~K}$ ) is 9 to 14 orders of magnitude slower than rotation of a single molecule (typically $\omega \sim 10^{9}$ to $10^{12} \mathrm{~s}^{-1}$ ). The angular velocity reaches 0 upon approach to the two dimensional liquid-to-solid transition in the monolayer at $T=285.8 \mathrm{~K}$. We estimate the rotational viscosity, $\gamma_{1}$, in the monolayer and the torque, $\Gamma$, driving this rotation. The torque per molecule equals $\Gamma=5.7 \times 10^{-8} \mathrm{pN} \mathrm{nm}$ at $310 \mathrm{~K}\left(\gamma_{1}=0.081 \mathrm{~Pa} \mathrm{~s}, \omega=0.87 \mathrm{~s}^{-1}\right)$. The energy generated during one turn of the molecule at the same temperature is $W=3.5 \times 10^{-28} \mathrm{~J}$. Surprisingly, although this energy is 7 orders of magnitude smaller than the thermal energy, $k_{\mathrm{B}} T(310 \mathrm{~K})=4.3 \times$ $10^{-21} \mathrm{~J}$, the rotation is very stable. The potential of the studied effect lies in the collective motion of many $\left(>10^{12}\right)$ "nano-windmills" acting "in concerto" at the scale of millimetres. Therefore, such systems are candidates for construction of artificial molecular engines, despite the small energy density per molecular volume ( 5 orders of magnitude smaller than for a single ATPase).

Received 8th July 2013

Accepted 12th July 2013

DOI: $10.1039 / \mathrm{c} 3 \mathrm{nr} 03496 \mathrm{~h}$

www.rsc.org/nanoscale low angular velocities (often $<10^{-3} \mathrm{~Hz}$ ) and it was a challenge to increase the speed of rotation up to 87 revolutions per second by a special design of the molecule. ${ }^{11}$ In this manuscript we show a mechanism of rotation powered by the unidirectional flux of water molecules in the angular velocity range of 1 to $10^{-2} \mathrm{~s}^{-1}$.

None of the papers dealing with AMMs considered the Lehmann effect, ${ }^{12}$ which describes the collective rotation of chiral molecules of a liquid crystal (LC) driven by a temperature gradient, as a possible mechanism driving the AMMs. Another possible mechanism for powering AMMs was discovered by Tabe and Yokoyama; ${ }^{13}$ here collective molecular precession occurs in Langmuir monolayers of chiral LC. This phenomenon can be considered as a chemical Lehmann effect because the collective molecular precession is driven by a vapour pressure difference, i.e. a gradient of the chemical potential. Detailed quantitative analysis of the driving forces in this phenomenon over a wide temperature range is the aim of our work. We also consider the following question: how does the chemical Lehmann effect compare with the biological mechanism in ATPase, which is the protein utilizing the gradient of proton concentration to drive the rotation and ADP-to-ATP synthesis?

The chemical Lehmann effect, after its discovery by Tabe and Yokoyama, ${ }^{13}$ was investigated experimentally in different aspects by us ${ }^{14}$ and by Gupta $e t a l .{ }^{15}$ The discussion presented here is based on the new measurements of the frequency of the collective molecular precession at different temperatures in the range of $285-312 \mathrm{~K}$. This study allowed us to estimate the rotational viscosity in the monolayer and the torque driving this 
rotation. The torque should be compared to the one generated by other AMMs and especially, by the working proteins among which the rotating ATPase ${ }^{\mathbf{1 6}}$ seems to be the best candidate. Detailed discussion on that subject is placed at the end of this paper.

The collective molecular rotation or precession mentioned above belongs to the broad class of phenomena called Onsager's cross-effects found in different branches of physics and chemistry, especially irreversible thermodynamics. The most known examples include the Hall effect, Marangoni effect, Soret effect (thermodiffusion), and many others, in which a flux of matter (mass or energy) is coupled with a thermodynamic force. A less known effect of this type is called by the name of Otto Lehmann, ${ }^{12}$ who observed rotation of droplets of cholesteric LC in a gradient of temperature and described it in 1900. Because of experimental difficulties the Lehmann's experiment was not reproduced for more than 100 years, although it was explained theoretically in 1968 by Leslie $^{17}$ and later in 1982 it was confirmed indirectly by Éber and Jánossy. ${ }^{18}$ Finally, in 2008 the Lehmann's observation was reproduced in the sophisticated experiments by Oswald et al. ${ }^{\mathbf{1 9 - 2 1}}$ Very recently Oswald summarized his investigations with emphasis on the microscopic and macroscopic origin of the Lehmann effect. ${ }^{22}$ Theoretical papers on that subject were also published by Brand et al..$^{23-25}$ to mention just a few examples of studies on this topic. An assumption that "inverse Lehmann effects can be used as a microscopic pump", given earlier by Svenšek et al. ${ }^{24}$ makes us think that such a mechanism could be involved in the working of $\mathrm{F}_{0}$-ATPase as a proton pump.

\section{Results and discussion}

\section{Molecular precession in Langmuir monolayers}

Here we present the results for just one liquid-crystalline ferroelectric compound: $(S) 4$-[4-(1-methylheptyloxycarbonyl)phenyl]-4'-[6-(cyanoetanoyloxy)hexyloxy]biphenyl-4-carboxylate, denoted as ferroC, although we studied more ferroelectric compounds and observed the collective rotations with similar behaviour for two of them. The molecules of both compounds self-assemble at the air-water interface and create monolayers of smectic-C* type. The polar molecules of LC are anchored to the water surface with the polar groups present at one end of each molecule. The asymmetric chiral groups attached to the other ends of the molecules' rigid cores stick out into the air. Projections of tilted molecules onto the plane of the water surface, often called a $c$-director, locally align along one preferred direction and create the specific patterns because of collective rotation driven by a flux of evaporating molecules of water. Molecules of water hitting the asymmetric branches of the chiral groups cause the resultant torque and the molecular precession. This mechanism of rotation was explained and proved by Tabe and Yokoyama. ${ }^{13}$ The rotation is not coupled to any macroscopic flow within the layer. The lack of flow in the monolayer becomes obvious after longer observation of few domains visible in the two images shown in Fig. 1 recorded using a Brewster angle microscope (BAM) with 8 seconds time delay. A gray-scale intensity of reflected polarized light, being in
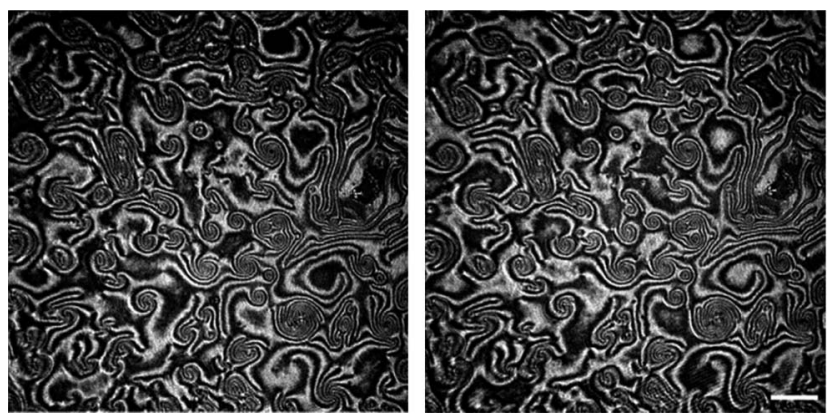

Fig. 1 Two images of the same area recorded at $25^{\circ} \mathrm{C}$ with 8 seconds of time delay showing that there is no flow in the film in spite of the rotation of the local ordering vector (the c-vector turns here approximately by $\pi / 2$ ); the patterns visible under a Brewster Angle Microscope (BAM) show that the shapes of domains remain unchanged and only the intensity of light reflected from bigger domains changes: bright regions turn black and vice versa; white bar shows a length of $500 \mu \mathrm{m}$.

correlation with the rotating director, changes from black to white and vice versa, but the shapes of the domains with uniform orientation remain unchanged (compare details of both images in Fig. 1). The image analysis of the recorded sequence of images allowed us to measure the frequency of rotation in a particular domain as explained in Fig. S1 in the ESI. $\uparrow$ This continuous collective rotation of the molecules is a result of the mechanical balance between the driving force causing a molecular precession and the viscous torque, $\gamma_{1}$, proportional to the rotational viscosity. Examples of typical patterns created during molecular precession in the Langmuir film and observed using BAM are shown in Fig. 1 and 2. The mechanism of the pattern formation was explained theoretically and through computer simulations by Svenšek et al. ${ }^{25}$ This mechanism results in a phase shift of the $c$-director and macroscopic "in-plane" spirals in the monolayer caused by a winding up process. Detailed analysis of many patterns recorded during our studies has shown clearly a time evolution of these patterns with winding-up causing a shortening of the pitch of spirals in the plane of the monolayer. It can be seen in Fig. 2 showing the early-stage (Fig. 2a) and the late-stage (Fig. 2b) of the pattern evolution. An interesting theoretical explanation of the origin of the patterns with macroscopic chirality of the monolayer, caused by rotation of the axial dynamic preferred direction, was published recently by Brand et $a{ }^{23}$ Another theoretical interpretation supported by
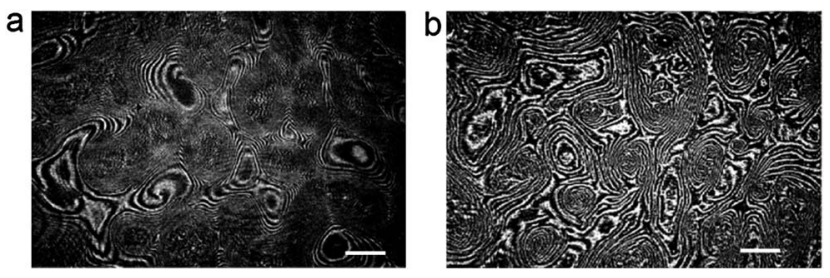

Fig. 2 Early (a) and late (b) stages of pattern evolution; image (b) shows stripes tightly "wound-up" around each center of rotation; the images were recorded with a time delay of 1.5 hour; white bars show a length of $500 \mu \mathrm{m}$ 
computer simulations of pattern formation was given (soon after the discovery of this phenomenon by Tabe and Yokoyama $^{13}$ ) by Tsori and deGennes. ${ }^{26}$ The authors assumed ${ }^{26}$ that the rotation is induced by sudden "bursts" of evaporation (when the threshold of the film elasticity is overcome by the difference of chemical potentials of water in a subphase and in a vapour), and is blocked at the domain walls. Images in Fig. 2 seem to support this model.

\section{Torque and rotational viscosity}

The surface torque, $\Gamma_{\mathrm{s}}$, being a driving force causing the rotation of the $c$-director in the monolayer, is defined as $\Gamma_{\mathrm{s}}=f r / A$, where $f$ is the force acting on the chiral group (treated as vanes of the propeller ${ }^{13}$ ), $r$ is the projection of the distance between the polar and the chiral groups on the plane of the water surface and $A$ is the molecular area, i.e. the area occupied by one molecule in the monolayer. Here $A$ is measured during the recording of Langmuir isotherms. Continuous precession of the molecule at constant angular velocity, $\omega$, is a result of the balance between the driving torque and the viscous torque, which can be written as

$$
\Gamma_{\mathrm{s}}=\gamma_{\mathrm{s}} \omega
$$

where $\gamma_{\mathrm{s}}$ is the surface viscosity. ${ }^{21,27}$ The surface viscosity is an important component that strongly affects the values of the rotational viscosity, usually measured in thin samples confined between two glass plates, but it cannot be measured directly in Langmuir monolayers. The surface viscosity has to be taken into account as a kind of correction to the measured values of the rotational viscosity $\gamma_{1}$, as explained in detail by Oswald. ${ }^{27}$ Both surface quantities, $\Gamma_{\mathrm{s}}$ and $\gamma_{\mathrm{s}}$, are related to the bulk properties through the relationships: $\Gamma=\Gamma_{\mathrm{s}} / d$ and $\gamma_{1}=\gamma_{\mathrm{s}} / d$, where $d$ is the thickness of the layer. The surface viscosity, $\gamma_{\mathrm{s}}$, as measured in a monolayer and the rotational viscosity, $\gamma_{1}$, measured in a bulk phase are the same physical properties after scaling through the relationship $\gamma_{1}=\gamma_{\mathrm{s}} / d$ because we consider just one interfacial layer interacting with water at the bottom and with air at the top. Our ferroelectric LC is anchored to the water surface with a very small cyano group, and the viscosity of the air on the other side is so small that the shear between the monolayer and both media (below and above) is negligible.

The bulk torque, $\Gamma$, is proportional to the momentum flux of evaporating water molecules. We estimate the flux from the Hertz-Knudsen relationship. This flux is proportional to the pressure of saturated water vapour, $p_{\text {sat }}$ (for details see ref. 28). In our case water evaporation occurs at a pressure difference $\Delta p=p_{\text {sat }}-p_{\mathrm{w}}$, where $p_{\mathrm{w}}$ is the partial pressure of water vapour in a humid atmosphere over the Langmuir film. Thus, the balance between the driving torque and the viscous torque can be written as:

$$
\Gamma=\nu \Delta p=\gamma_{1} \omega
$$

where $\nu$ is the chemical Lehmann coefficient, $\Delta p=p_{\text {sat }}-p_{\mathrm{w}}$ is explained above, $\gamma_{1}$ is the rotational viscosity, and $\omega=\mathrm{d} \varphi / \mathrm{d} t$ is the angular velocity. The chemical Lehmann coefficient, $\nu$, is a material constant dependent mainly on the chiral strength of the chiral molecule, which is here understood intuitively as a parameter similar to the Lehmann rotatory power (LRP) defined for bulk chiral phases. ${ }^{29}$

The last quantity needed to estimate the surface torque and to compare our molecular rotors with biological engines is the area per molecule, $A$. In our experiments we determined $A$ at the air-water interface for ferroC by the Langmuir trough method. The compound ferroc, when spread from chloroform solution on the water surface, forms a very stable monolayer. The examples of two isotherms of the surface pressure, $\pi$, as a function of area per molecule, $A$, as well as the molecular structure of compound ferroC are given in Fig. 3. In the range of surface pressure, $\pi$, accessible on the water surface, the monolayer exhibits 2D transitions between gas, liquid, and solid phases. The morphology of the monolayer was observed using BAM. In Fig. 3, the range of molecular area $A>0.42 \mathrm{~nm}^{2}$ is the region of coexistence of gas and liquid phases. The range marked as 1 with the first pressure increase corresponds to a compression of the compact rotating liquid phase characterized by typical textures shown in Fig. 1 and 2 . With our setup it was impossible to distinguish between $\mathbf{n}$ and $-\mathbf{n}$ orientation of the ordering vector, so the grey-scale intensity variation from black to white and back to black means a rotation by angle $\pi$. At the beginning of the plateau (range 2) the phase transition to solid phase occurs. The plateau region corresponds to the liquidsolid phase transition accompanied by the reorientation of the molecules from tilted to vertical orientation (this observation comes from surface potential measurements ${ }^{30}$ not reported
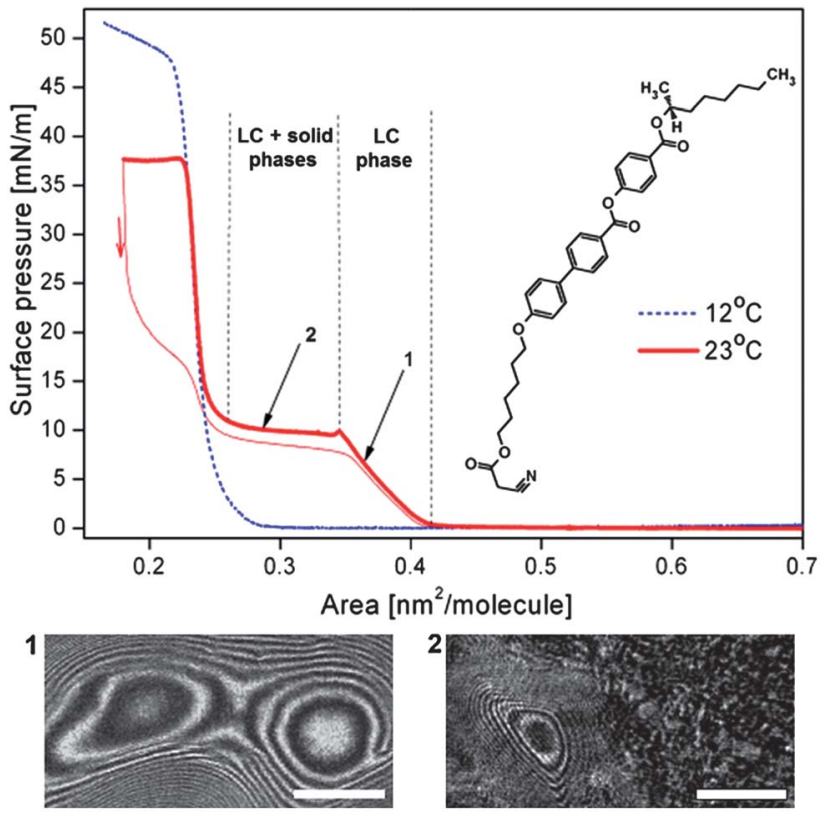

Fig. 3 Two isotherms of surface pressure vs. area per molecule for the ferroelectric liquid crystal of a given structure at the air-water interface at temperatures 285.2 and $296.2 \mathrm{~K}\left(12\right.$ and $\left.23^{\circ} \mathrm{C}\right)$; two BAM images show: (1) texture of the rotating domain recorded at point 1 ; (2) texture of the two-phase region observed at the plateau of the isotherm at $296.2 \mathrm{~K}\left(23^{\circ} \mathrm{C}\right)$. White bar shows a length of $500 \mu \mathrm{m}$. 
here). On further compression, the second pressure increase, corresponding to a homogenous solid phase, is observed and finally, at the second plateau at $\pi=38 \mathrm{mN} \mathrm{m}^{-1}$, the monolayer collapses with formation of random aggregates of the solid phase (see Fig. S2 in the ESI $\dagger$ ).

The coherent rotary motion of the $c$-director gave a characteristic oscillatory pattern of the reflected light observed under a BAM. The rate of rotation was examined at different temperatures of the water subphase in the range of 285-312 $\mathrm{K}$ and the results are shown in Fig. 4. The slowest possible to measure rotation (approx. 500 seconds for rotation by $2 \pi$ angle) was measured at temperature $285.8 \mathrm{~K}$. Below this temperature the oscillatory pattern of the rotating liquid phase vanishes in the transition to the solid phase (see Fig. S3 in the ESI $\dagger$ ). We suppose that the diverging time of collective precession of chiral molecules and the decrease of macroscopic chirality of the monolayer support the theoretical model of Brand et al. ${ }^{23} \mathrm{We}$ show in Fig. 4 that the time of molecular revolutions increases with the decreasing temperature. Specifically, the rotation is slowing down from 6 seconds at $312.2 \mathrm{~K}$ to almost 8 minutes at $285.8 \mathrm{~K}$.

Spreading the monolayer of our compound on the water surface at temperature $285 \mathrm{~K}$, even at low concentrations, does not give a uniform 2D gas phase. Instead, big rafts of the solid phase are visible in BAM images. In the proximity of the transition to the solid phase (at $285.8 \mathrm{~K}$ ) the period of the revolution of the vector order parameter diverges. This slowing down of the rotation of the $c$-vector corresponds to the divergence of the rotational viscosity.

We analyzed the data of $\omega(T)$ using eqn (1) with $\Delta p$ substituted by $p_{\text {sat }}$ because all the measurements were performed in an atmosphere of dry argon, thus $p_{\mathrm{w}}=0$. In the equation:

$$
\omega=\frac{\nu p_{\text {sat }}}{\gamma_{1}}
$$

the variables $p_{\text {sat }}$ and $\gamma_{1}$ have to be replaced by their theoretical dependence on the temperature. The saturated vapour pressure of water, $p_{\text {sat }}$, as a function of temperature was replaced by eqn

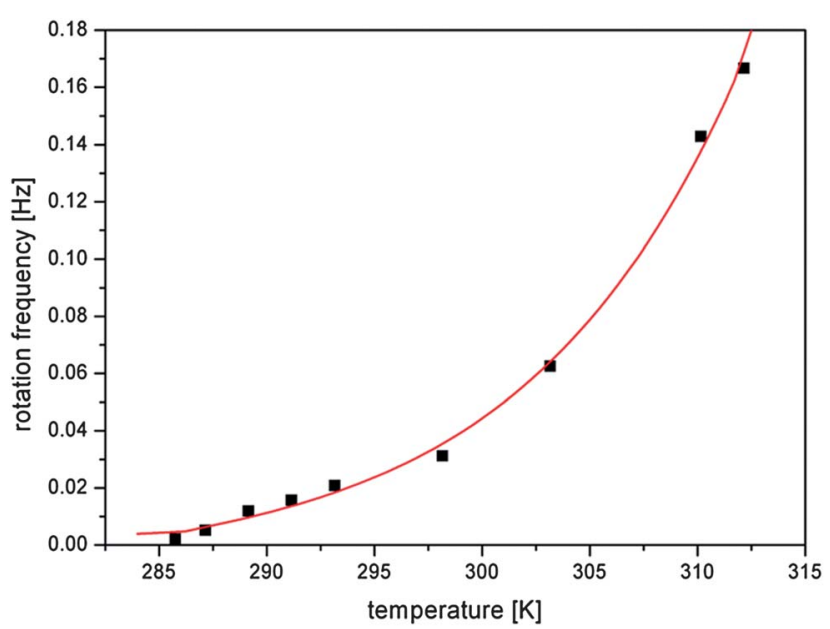

Fig. 4 Frequency of rotation by $2 \pi$ angle as a function of temperature of the water subphase (the line is an exponential fit used for data interpolation).
(3) suggested by the National Institute of Standards and Technology ${ }^{31}$ as the most precise in the temperature range we are interested in. The equation has the form:

$$
\ln p=16.16629-3736.276 /(T-49.577)
$$

where $p$ is expressed in $\mathrm{kPa}$, and $T$ is expressed in $\mathrm{K}$. In liquid crystalline phases, the rotational viscosity, in general, depends on the temperature through rotational diffusion coefficient and orientational order parameters. ${ }^{32}$ Here, for temperature dependence of the rotational viscosity, $\gamma_{1}(T)$, we applied the well known Vogel-Fulcher-Tammann ${ }^{33}$ model (VFT), commonly used to describe the bulk viscosity of glass forming liquids close to the glass transition. In the case of the monolayer we did not observe the real glass transition, which usually occurs in a metastable state below the temperature of solidification. For that reason, we introduced in the exponent of the VFT equation the additional term $\left(T-T_{\mathrm{c}}\right)$ that contains information about the temperature of the transition to the solid phase, $T_{\mathrm{c}}$. The equation used in further analysis reads

$$
\gamma_{1}=\gamma_{0} \exp \left(\frac{B\left(T-T_{\mathrm{c}}\right)}{T-T_{0}}\right),
$$

where $\gamma_{0}$ is a material dependent parameter, $B$ is a dimensionless parameter, $T_{0}$ is the asymptotic temperature at which the viscosity diverges, and $T_{\mathrm{c}}$ is the experimentally established temperature for the phase transition to the solid state.

With the values of $p_{\text {sat }}$ calculated for each temperature from eqn (3), we calculated from eqn (2) the values of the ratio $\gamma_{1} / \nu=$ $p_{\text {sat }} / \omega$ as a function of temperature. These values behave the same way as the rotational viscosity because $\nu$ is a constant. We fitted the values of $\gamma_{1} / \nu$ as a function of temperature using the VFT model given by eqn (4) with an experimental value of $T_{\mathrm{c}}=$ $285.0 \mathrm{~K}$ and treating $\gamma_{0}, B$ and $T_{0}$ as adjustable parameters.

This fitting to eqn (4) gave very reasonable values of the parameters $B=-3.564 \pm 0.138$ and $T_{0}=(282.16 \pm 0.55) \mathrm{K}$. It has to be mentioned that these values are not affected by the multiplication factor $\nu$. The real values of temperature dependence of $\gamma_{1}$ (still with accuracy to the multiplication factor) were found by scaling the measured ratios of $\gamma_{1} / \nu$ to typical values of the rotational viscosity of smectic-C* phases in ferroelectric LCs far from any phase transition. For scaling we chose the average value of $\gamma_{1}=0.1 \mathrm{~Pa}$ s found in the literature ${ }^{34,35}$ for ferroelectric LCs at a temperature in the middle of the range of $\mathrm{SmC}^{*}$ (between the transitions to solid and to SmA phases). The value of $\gamma_{1}=0.1$ Pa s was taken as the value of the rotational viscosity of the monolayer of compound ferroC at temperature $T=300 \mathrm{~K}$. This scaling and fitting of $\gamma_{1}(T)$ to eqn (4) gave the value of parameter $\gamma_{0}=(2.00 \pm 0.23)$ Pa s. Final results of fitting and the fitted curve are shown in Fig. 5. Above scaling allowed us to estimate the chemical Lehmann coefficient, $\nu$, and gave the value $\nu=8.8 \times 10^{-6}$. In this case $\nu$ is a dimensionless parameter related to the chiral strength of the molecule characterized by the Lehmann Rotatory Power ${ }^{29}$ (LRP). Untill now, there has been no theoretical prediction on how the chemical Lehman coefficient relates to the classic Lehmann coefficient, which controls the molecular rotation in a temperature gradient. Very recent 




Fig. 5 Rotational viscosity, $\gamma_{1}$, of the monolayer and the output power of the single molecule vs. temperature of the subphase; the points marked by triangles $(\boldsymbol{\Lambda})$ are calculated from the angular velocity (see text); the solid curve is a result of fitting to eqn (4) (the equation is also given in the graph together with fitted parameters); dots ( ) represent the power output from one molecule calculated for experimental points; the dashed curve is calculated according to eqn (7).

measurements by Oswald ${ }^{22}$ gave the value equal to $2.7 \times 10^{-7}$ $\mathrm{N} \mathrm{m}^{-1} \mathrm{~K}^{-1}$. Both of these coefficients are related to the chiral strength of the molecule or LRP, so the relationship between them should also exist.

\section{Ferroelectric LC and ATPase: two monomolecular machines}

We estimated the viscous torque, $\Gamma_{\mathrm{fC}}$, of the ferroC monolayer from calculated values of $\gamma_{1}$. This quantity is equal to the torque of unit volume, according to eqn (1) and measured in $\mathrm{N} \mathrm{m}^{-2}$ unit. For the single-molecule the torque is usually measured in $\mathrm{pN}$ nm. To estimate the torque created by a single rotating molecule of ferroC, the viscous torque has to be multiplied by the volume occupied by one molecule. We calculate these values of torque for different temperatures as:

$$
q_{\mathrm{fC}}=\Gamma_{\mathrm{fC}} A_{\mathrm{fC}} d=\gamma_{1} \omega A_{\mathrm{fC}} d,
$$

where $q_{\mathrm{fC}}$ is the torque of a single molecule of ferroC, $\Gamma_{\mathrm{fC}}$ is the viscous torque of the monolayer, $A_{\mathrm{fC}} \approx 0.4 \mathrm{~nm}^{2}$ is the area occupied by one molecule in the monolayer as estimated from the isotherm in Fig. 3, and $d=2.0 \mathrm{~nm}$ is the thickness of the monolayer of ferroc. ${ }^{30}$ The torque of a single molecule, $q_{\mathrm{fC}}$, estimated from eqn (5), using smoothed data of $\gamma_{1}$ and $\omega$ at temperature $310 \mathrm{~K},\left(\gamma_{1}=0.081 \mathrm{~Pa} \mathrm{~s}, \omega=0.87 \mathrm{~s}^{-1}\right)$ equals $5.7 \times$ $10^{-8} \mathrm{pN} \mathrm{nm}$. We estimate the output work from a single molecule generated during one revolution, $W$, and the power of such a monomolecular machine, $P$, from the following equations:

$$
W=2 \pi q_{\mathrm{fC}}=2 \pi \gamma_{1} \omega A_{\mathrm{fC}} d
$$

and

$$
P=W \omega / 2 \pi=\gamma_{1} \omega^{2} A_{\mathrm{fC}} d
$$

The energy and power generated during one turn of the molecule calculated from eqn (6) and (7) at $T=310 \mathrm{~K}$ are $W=$
$3.5 \times 10^{-28} \mathrm{~J}$ and $P=4.9 \times 10^{-29} \mathrm{~W}$, respectively. The values of the power of one molecule calculated for experimental points are shown in Fig. 5 together with the curve $P(T)$ calculated from eqn (7) and smoothed data of $\gamma_{1}(T)$ and $\omega(T)$. In spite of the fact that the energy equivalent to work estimated above is 7 orders of magnitude smaller than $k_{\mathrm{B}} T(310 \mathrm{~K})=4.3 \times 10^{-21} \mathrm{~J}$, the patterns observed in the film are not destroyed by thermal motion because of the collectiveness of the molecular precession and interactions of the monolayer film with the water surface.

We can compare our result of the torque per molecule, $q_{\mathrm{fC}}=$ $5.7 \times 10^{-8} \mathrm{pN} \mathrm{nm}=5.7 \times 10^{-29} \mathrm{~J}$, to the value of torque estimated by Tabe and Yokoyama ${ }^{\mathbf{1 3}}$ for their chiral molecule rotating in the monolayer. They report the value of $q_{\mathrm{T \& Y}}=\left(10^{-11}\right.$ to $\left.10^{-10}\right) k_{\mathrm{B}} T<4.3 \times 10^{-31} \mathrm{~J}$, approximately 100 times less than our value. The difference is understandable because Tabe and Yokoyama studied molecules of different shapes, although of similar size, in the monolayer spread on the mixture of water and glycerol (more viscous subphase). Also the method of torque estimation was different because their calculation was based on the elasticity of the monolayer and the size of the domain in which the collective precession occurs. Nevertheless, both results drive to similar conclusions given in the next section of this paper.

None of the parameters estimated above for ferroc can be compared directly to the data accessible in the literature for ATPase. The complex of $\mathrm{F}_{0} \mathrm{~F}_{1}-\mathrm{ATPase}$ has been investigated by many authors.,.$^{3,36-38}$ The reported values of torque of ATPase, $q_{\mathrm{ATP}}$, ranges from $10 \mathrm{pN}$ nm to $80 \mathrm{pN}$ nm depending on the load connected to the ATPase rotor and the frequency of rotation. ${ }^{37-40}$ The mechanism of rotation is different in ferroC and in ATPase. The latter is very clearly explained by Aksimentiev et al. ${ }^{\mathbf{4 1}}$ The coupled units of $\mathrm{F}_{0}$-ATPase and $\mathrm{F}_{1}$-ATPase alternately exchange their roles and when $\mathrm{F}_{1}$ plays the role of a motor, driven by ATP hydrolysis, then $\mathrm{F}_{0}$ acts as a pump generating the proton gradient. In the alternative case, the $\mathrm{F}_{0}$ unit acts as a motor driven by the gradient of protons and $F_{1}$ synthesizes ATP. Aksimentiev et al. $^{\mathbf{4 1}}$ reported the results of sophisticated simulations of the system of $\mathrm{F}_{0}$ unit, consisting of more than 111 thousands of atoms, giving the volume of this unit equal to $11.2 \times 12.3 \times 9.8 \mathrm{~nm}^{3}$. Similar dimensions of the cross-section of the complex of $\mathrm{F}_{0} \mathrm{~F}_{1}-\mathrm{ATPase}$ are reported by Stock et al. ${ }^{42}$ with the height of the complex equal to $19.1 \mathrm{~nm}$. We relate the energy output of our "ferroC molecular motor" to much bigger $\mathrm{F}_{0} \mathrm{~F}_{1}$ ATPase taking into account differences in their molecular volumes. We calculate the energy density, $\varepsilon$, dissipated during one revolution of a single molecule as a ratio of the output work of one revolution divided by the volume, $V$, of one molecule,

$$
\varepsilon=W / V=2 \pi q / V=2 \pi \gamma_{1} \omega
$$

The volume of the ferroC molecule has already been estimated, so in this case $\varepsilon_{\mathrm{fC}}=2 \pi \Gamma_{\mathrm{fC}}=0.44 \mathrm{~N} \mathrm{~m}^{-2}=4.4 \times 10^{-7} \mathrm{pN}$ $\mathrm{nm}^{-2}$. The volume of $\mathrm{F}_{0} \mathrm{~F}_{1}-\mathrm{ATPase}$, calculated according to its dimensions from ref. 41 and 42 , is equal to $2630 \mathrm{~nm}^{3}$. By using the most frequently reported value of the torque $\mathrm{in}^{\mathbf{4 1 3}}$ of ATPase equal to $40 \mathrm{pN} \mathrm{nm}$, we obtain the energy density $\varepsilon_{\mathrm{ATP}}=1.5 \times 10^{-2}$ 
$\mathrm{pN} \mathrm{nm}{ }^{-2}$. Such a comparison of the output energy density, $\varepsilon$, that could be generated by a biological motor, such as ATPase, and by an artificial molecular motor, such as collectively rotating chiral molecules of LC, gives the ratio of energy density equal to $10^{5}$. Thus, the work per unit molecular volume performed by ATPase driven by ATP hydrolysis is 100000 times greater than that performed by our ferroC molecule driven by the flux of water molecules. This comparison shows that the future of artificial engines belongs to biological units, possibly working in other environment than living systems. However, the big advantage of AMMs based on liquid crystalline molecules is their simplicity and the easily tuneable, collective rotation involving $10^{12}$ molecules. A perfect high energy density machine would comprise these two concepts of collective behaviour (such as in the case of our "nano-windmills") with a very efficient single entity unit (such as ATPase).

\section{Concluding remarks}

In summary, the evaporation of water across a chiral liquid crystalline monolayer can drive a collective molecular precession. ${ }^{\mathbf{1 3 , 1 4}}$ We have shown that in Langmuir monolayers the angular velocity of rotation can be extremely low (of the order of $10^{-2} \mathrm{~Hz}$ ), i.e. 14 orders of magnitude lower than that of typical molecular rotations. This unusual slowing down of collective unidirectional rotation is caused by the viscosity of the monolayer and the proximity of the two dimensional liquid-solid phase transition in the monolayer of the smectic$\mathrm{C}^{*}$ phase. We have determined the temperature dependence of the rotational viscosity, $\gamma_{1}$, from BAM observations of molecular precession in Langmuir films. The experimental study of temperature dependence of the rotational viscosity, $\gamma_{1}(T)$, of Langmuir monolayers facilitated estimation of the torque engendered by one rotating molecule. We have related the energy density output of this "molecular motor" to the highly efficient biological motor $\mathrm{F}_{0} \mathrm{~F}_{1}$-ATPase. Referring to the title of the paper, it can be concluded that the Lehmann rotation has potential to be used to power a "nano-windmill", however, the power of such an "engine" is 5 orders of magnitude smaller than the power of a single biological motor. But, the potential of the Lehmann effect is hidden in the collective nature of motion of many $\left(>10^{12}\right)$ "nano-windmills" acting "in concerto" at the scale of millimetres. We expect that future artificial molecular machines should combine efficient biological units synchronized by a kind of Lehmann effect, utilizing concentration or temperature gradients, and perform their function collectively.

\section{Acknowledgements}

$\mathrm{RH}$ thanks the National Science Centre for funding the project from the funds granted on the basis of the decision number: 2011/02/A/ST3/00143 (Maestro grant). MF acknowledges the financial support from the Foundation for Polish Science Team Programme TEAM/2010-6/4 co-financed by the EU "European Regional Development Fund".

\section{Notes and references}

1 K. Kinosita, R. Yasuda, H. Noji, S. I. Ishiwata and M. Yoshida, Cell, 1998, 93, 21-24.

2 G. Oster and H. Wang, Structure, 1999, 7, R67-R72.

3 K. Kinbara and T. Aida, Chem. Rev., 2005, 105, 1377-1400.

4 J. Michl and E. C. H. Sykes, ACS Nano, 2009, 3, 1042-1048.

5 E. R. Kay, D. A. Leigh and F. Zerbetto, Angew. Chem., Int. Ed., 2007, 46, 72-191.

6 A. Coskun, M. Banaszak, R. D. Astumian, J. F. Stoddart and B. A. Grzybowski, Chem. Soc. Rev., 2012, 41, 19-30.

7 M. Klok, N. Boyle, M. T. Pryce, A. Meetsma, W. R. Browne and B. L. Feringa, J. Am. Chem. Soc., 2008, 130, 10484-10485.

8 N. Koumura, R. W. J. Zijlstra, R. A. van Delden, N. Harada and B. L. Feringa, Nature, 1999, 401, 152-155.

9 A. Harada, J. Li and M. Kamachi, Nature, 1992, 356, 325-327. 10 A. Schalley, K. Beizai and F. Vögtle, Acc. Chem. Res., 2001, 34, 465-476.

11 J. Vicario, M. Walko, A. Meetsma and B. L. Feringa, J. Am. Chem. Soc., 2006, 128, 5127-5135.

12 O. Lehmann, Ann. Phys., 1900, 2, 649-705.

13 Y. Tabe and H. Yokoyama, Nat. Mater., 2003, 2, 806-809.

14 P. Milczarczyk-Piwowarczyk, A. Żywociński, K. Noworyta and R. Hołyst, Langmuir, 2008, 24, 12354-12363.

15 R. K. Gupta, K. A. Suresh, S. Kumar, L. M. Lopatina, R. L. R. Selinger and J. V. Selinger, Phys. Rev. E: Stat., Nonlinear, Soft Matter Phys., 2008, 78, 041703.

16 H. Noji, R. Yasuda, M. Yoshida and K. Kinosita, Nature, 1997, 386, 299-302.

17 F. M. Leslie, Proc. R. Soc. London, Ser. A, 1968, 307, 359-372.

18 N. Éber and I. Jánossy, Mol. Cryst. Liq. Cryst., 1982, 72, 233238.

19 P. Oswald and A. Dequidt, Phys. Rev. Lett., 2008, 100, 217802.

20 A. Dequidt, A. Żywociński and P. Oswald, Eur. Phys. J. E, 2008, 25, 277-289.

21 P. Oswald, A. Dequidt and A. Żywociński, Phys. Rev. E: Stat., Nonlinear, Soft Matter Phys., 2008, 77, 061703.

22 P. Oswald, Eur. Phys. J. E, 2012, 35, 10, and references therein.

23 H. B. Brand, H. Pleiner and D. Svenšek, Eur. Phys. J. E, 2011, 34, 1-10.

24 D. Svenšek, H. Pleiner and H. R. Brand, Phys. Rev. E: Stat., Nonlinear, Soft Matter Phys., 2008, 78, 021703.

25 D. Svenšek, H. Pleiner and H. R. Brand, Phys. Rev. Lett., 2006, 96, 140601.

26 Y. Tsori and P. G. de Gennes, Eur. Phys. J. E, 2004, 14, 91-96. 27 P. Oswald, G. Poy, F. Vittoz and V. Popa-Nita, Liq. Cryst., 2013, 40, 734-744.

28 R. Hołyst and M. Litniewski, J. Chem. Phys., 2009, 130, 074707.

29 P. Oswald, L. Jụrgensen and A. Żywociński, Liq. Cryst., 2011, 38, 601-613.

30 P. Nitoń, Langmuir monolayers of ferroelectric and bolaamphiphilic liquid crystal at the air-water interface, Ph. D. thesis, IPCh PAS, Warsaw, 2012.

$31 \mathrm{~W}$. Wagner and A. Pruss, International Equations for the Saturation Properties of Ordinary Water Substance. 
Revised According to the International Temperature Scale of 1990. Addendum to J. Phys. Chem. Ref. Data 16, 893 (1987), J. Phys. Chem. Ref. Data, 1993, 22, 783-787.

32 M. Fiałkowski, Phys. Rev. E: Stat. Phys., Plasmas, Fluids, Relat. Interdiscip. Top., 1998, 58, 1955-1966.

33 J. C. Mauro, Y. Yue, A. J. Ellison, P. K. Gupta and D. C. Allan, Proc. Natl. Acad. Sci. U. S. A., 2009, 106, 19780-19784.

34 C. Escher, T. Geelhaar and E. Bohm, Liq. Cryst., 1988, 3, 469-484.

35 W. Piecek, J. Rutkowska, J. Kędzierski, P. Perkowski, Z. Raszewski and R. S. Dąbrowski, Measurements of Rotational Viscosity of SmC $^{*}$ Mixtures, in Liquid Crystals; International Society for Optics and Photonics, 1998, pp. 148-153.

36 R. Yasuda, H. Noji, M. Yoshida, K. Kinosita Jr and H. Itoh, Nature, 2001, 410, 898-904.
37 T. Bilyard, M. Nakanishi-Matsui, B. C. Steel, T. Pilizota, A. L. Nord, H. Hosokawa and R. M. Berry, Philos. Trans. $R$. Soc., B, 2013, 368, 1611.

38 W. Junge, H. Sielaff and S. Engelbrecht, Nature, 2009, 459, 364-370.

39 H. Omote, N. Sambonmatsu, K. Saito, Y. Sambongi, A. Iwamoto-Kihara, T. Yanagida and M. Futai, Proc. Natl. Acad. Sci. U. S. A., 1999, 96, 7780-7784.

40 O. Pänke, D. A. Cherepanov, K. Gumbiowski, S. Engelbrecht and W. Junge, Biophys. J., 2001, 81, 1220-1233.

41 A. Aksimentiev, I. A. Balabin, R. H. Fillingame and K. Schulten, Biophys. J., 2004, 86, 1332-1344.

42 D. Stock, A. G. Leslie and J. E. Walker, Science, 1999, 286, 1700-1705.

43 T. Ariga, T. Masaike, H. Noji and M. Yoshida, J. Biol. Chem., 2002, 277, 24870-24874. 